\title{
DISTRIBUTION OF JAUNDICE IN CIRCULATORY FAILURE ${ }^{1}$
}

\author{
By JONATHAN MEAKINS \\ (From the Department of Medicine, McGill University Clinic, Royal Victoria Hospital, \\ Montreal)
}

(Received for publication, November 14, 1926)

The appearance of biliary pigmentation (jaundice) of the skin and viscera is a relatively common occurrence in severe circulatory failure. It has usually been considered that the distribution of this pigmentation was of a general character. A case was reported in 1925 (1) which showed that this was not always true and since then a number of similar cases have come under examination which have demonstrated that a definite localization of the pigmentation may occur under certain conditions of circulatory failure.

Case 1. A man with chronic valvular disease of the heart; (aortic stenosis and regurgitation, mitral regurgitation), tricuspid insufficiency, pulsating liver, myocardial failure, dependent anasarca and localized jaundice; death six days after admission.2

History. A white man, aged 37, with a history of recurring attacks of acute rheumatic fever and circulatory insufficiency. On December 15, 1924, the dyspnea suddenly became more troublesome and edema of the feet and legs rapidly appeared. On December 20th jaundice of the face, upper part of the body and sclerae appeared.

On examination on February 1, 1925, he was found to have the signs of aortic and mitral disease and pronounced evidence of tricuspid insufficiency with positive pulsation of the veins of the neck and pulsation of the liver which were both palpable and visible. There was edema of the feet, legs, genitalia, back and abdomen up to the level of the nipples (fig. 1). Over this area the skin was white without pigmentation while above this line the jaundice was an intense yellow color. Blood pressure was $138 \mathrm{~mm}$. Hg. systolic and $48 \mathrm{~mm}$. Hg. diastolic. There was periodic breathing and there were many fine moist râles at the bases of both

${ }^{1}$ Presented in abstract before the American Society for Clinical Investigation, May 3rd, 1926.

2 This case was reported in the Canadian Medical Association Journal, 1925, xv, 402. 
lungs. There was little sputum not blood-stained. The urine contained albumin, bile pigments and many casts. The blood showed a moderate nitrogen retention; the Wassermann reaction was negative; the van den Bergh test showed a strong direct and indirect reaction. The electrocardiogram revealed a regular sinus thythm with the auriculo-ventricular conduction time delayed ( 0.23 second); intraventricular conduction time was also delayed with the " $T$ " wave opposite to the main deflection in each lead, suggestive of a right bundle branch lesion. The edema fluid from the leg gave a negative direct and a very faint indirect van den Bergh reaction. The patient died six days after admission.

Necropsy findings. Productive and sclerotic endocarditis of aortic valve with stenosis and insufficiency; productive endocarditis involving the mitral valve and its chordae tendinae; hypertrophy and dilatation of the heart; productive pericarditis; productive pleurisy; red infarct of the lung, and advanced venous stasis of the liver.

Case 2. A man with auricular fibrillation, mitral regurgitation and stenosis, hypertrophy of the heart, severe myocardial failure with generalized dependent anasarca and jaundice of the upper part of the body. Died.

History. A white male, aged 33, who had been admitted to the Hospital on two previous occasions and gave a history of recurring attacks of circulatory failure but no history of rheumatic or syphilitic infection. Since his discharge from Hospital on December 3, 1924, he had remained in fairly good health until about the middle of May, 1925, when he noticed swelling of the feet, particularly at night. This gradually became worse and he suffered from severe dyspnea and palpitation on exertion. Then short periods of paroxysmal coughing developed. The edema progressed until it extended to the back and abdominal wall.

On examination on August 17, 1925, he was found to have signs of mitral stenosis with regurgitation and auricular fibrillation. There was pronounced edema in the feet, legs, back and abdominal wall. The lungs showed fine crepitations at the end of inspiration at the base of the left lung; the respirations were regular and he suffered from orthopnea. The blood Wassermann reaction was negative and there was slight nitrogen retention. The liver was palpable but not pulsating and there was no evidence of pulsation in the veins of the neck.

The patient's condition continued with slight periods of exacerbation and remission for many weeks.

Early in December, 1925, there was a very pronounced increase in his symptoms. The heart rate was between 140 and 160 per minute and further digitalis therapy was considered inadvisable on account of evidences of myocardial intoxication as demonstrated by the electrodardiograph. On December 5th acute symptoms of tricuspid insufficiency developed, with positive pulsation of the veins of the neck and pulsation of the liver. The edema had increased to a pronounced degree. On the 7th of December jaundice developed in the upper part of the body above the line of the edema including the face and the sclera. There was edema of the 


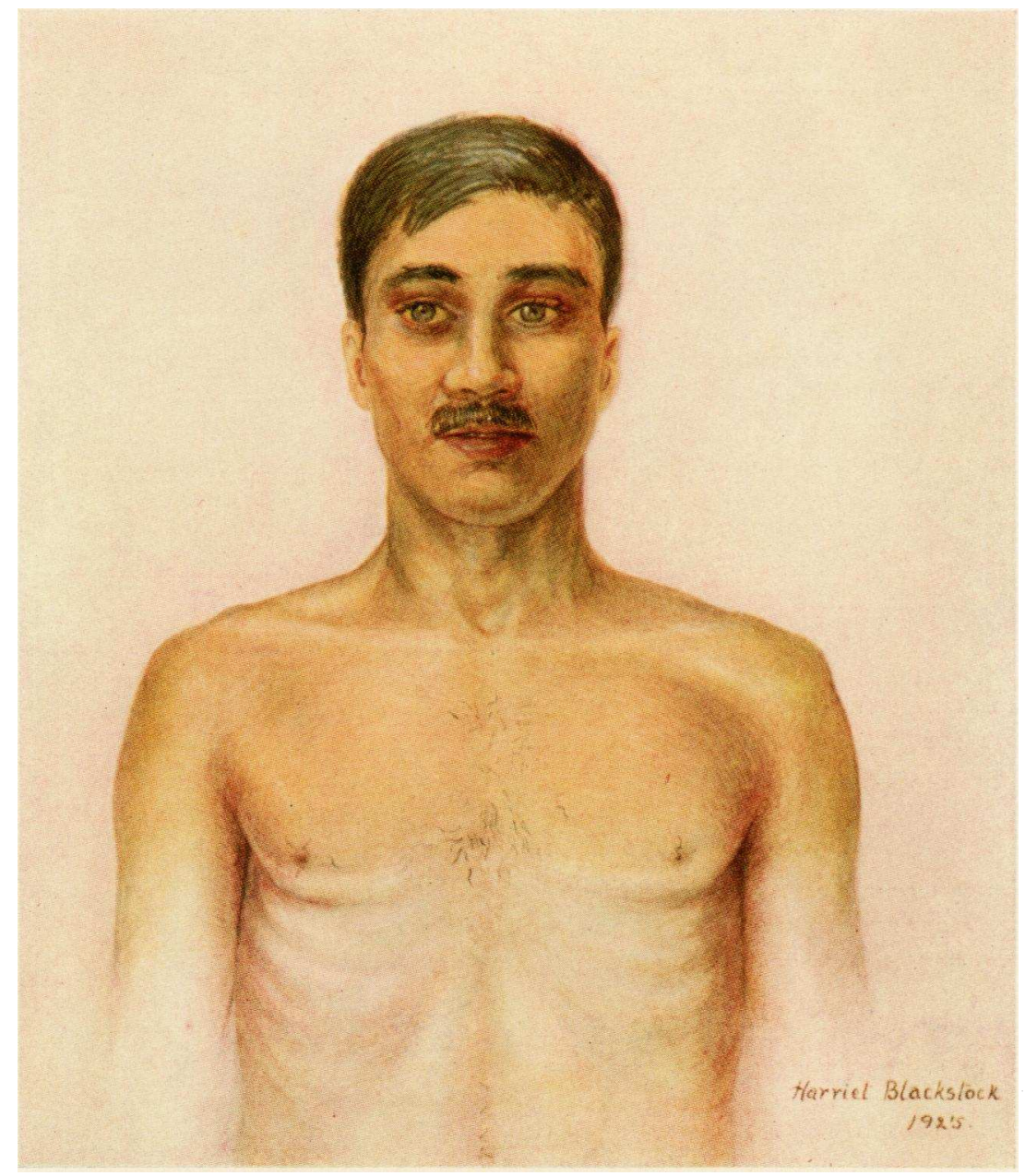

Fig. 1. Case 1. Showing Distribution of Jaundice in a Typical Case Here REPORTED 
arms to the insertion of the deltoid muscles. Over the edematous area no pigmentation was visible. Examination of the blood showed pronounced direct and indirect van den Bergh reactions and there was bile in the urine. On examination the edema fluid drawn from the legs was not pigmented and gave no positive van den Bergh reactions. Southey's tubes were inserted in both legs and many liters of fluid were withdrawn by this means. But in spite of the icterus becoming more and more intense in the upper parts of the body it did not appear over the edematous areas and it was only when the edema had practically disappeared locally around the site of the insertion of the Southey's tubes that very slight direct and indirect van den Bergh reactions were obtained. The patient died on December $12,1925$.

Necropsy findings. The necropsy findings were hypertrophy and dilatation of the heart; infarction of both lungs; hydropericardium; ascites and marked venous stasis of the liver.

Case 3. A woman with chronic syphilitic myocarditis, chronic mitral valvular insufficiency, auricular fibrillation, circulatory failure, general dependent anasarca and jaundice of the upper part of the body. Recovered.

History. A white woman, aged 50, who had been admitted to the Hospital six times since March, 1923, the last admission being on April 20, 1925. Since her discharge from Hospital on April 29, 1925, she was able to do but little owing to urgent dyspnea on exertion, with a certain amount of edema of the feet and legs in the evening. On July 6th she suddenly became conscious of the irregular and rapid cardiac rhythm, the dyspnea became more intense and she noticed a pulsating vein on the right side of her neck. The edema of the legs became rapidly worse, extending to the abdomen.

On examination on July 15th the patient appeared to be in extremis. The dyspnea was urgent and there was severe precordial pain. The feet, legs, abdominal wall and back were edematous to the nipple line and there was considerable ascites, some cyanosis of the lips and finger tips. There was evidence of fluid in both pleural cavities and the heart showed a definite systolic thrill at the apex beat which was $16 \mathrm{~cm}$. to the left of the mid-sternal line. There was a loud systolic murmur heard both at the apical and tricuspid areas. There was positive: pulsation of the right jugular vein and there was pronounced pulsation of the liver. There was jaundice of the face, sclerae and upper part of the body. The electrocardiogram revealed auricular fibrillation. On examination of the blood there was a positive Wassermann reaction and the direct and indirect van den Bergh reactions were both positive. The urine contained bile pigments but an examination of the ascitic fluid failed to show the presence of bile pigments as determined by the van den Bergh reactions.

Under treatment the patient made a rapid recovery. The signs of tricuspid insufficiency and jaundice disappeared while the edema subsequently became much less. 
Case 4. A man with severe myocardial failure, mitral insufficiency, aortic insufficiency (?), dependent edema and jaundice of the upper part of the body. Died.

History. A white man, aged 40 , who had been admitted to the Hospital on two previous occasions suffering from circulatory failure. After discharge from Hospital on October 15, 1925, he continued in fairly good health. Three days before admission (December 13,1925) he developed edema of the legs, the shortness of breath became exaggerated, he developed orthopnea and was confined to bed.

On examination the patient was found to have signs of circulatory failure with mitral insufficiency. The diagnosis of aortic insufficiency was in some doubt. Electrocardiogram showed a regular rhythm, 90 per minute; auriculo-ventricular conduction time 0.245 second. Examination of the blood showed a slight nitrogen retention, and the Wassermann reaction was negative. There was pronounced dependent anasarca extending to the nipple line, no edema of the arms. The liver was palpable but not pulsating.

His condition remained unchanged until January 17, 1926, when he suddenly developed pronounced dyspnea with precordial distress. He was found then to be suffering from acute tricuspid insufficiency as shown by pulsation of the veins of the neck and pulsation of the liver. Two days later there was jaundice of the upper part of the body above the line of edema, the face and the sclerae, while the edema of the dependent parts had much increased. Bile appeared in the urine and both the direct and indirect van den Bergh reactions in the blood were positive. The edema fluid showed no direct or indirect van den Bergh reaction. The patient died on January 23, 1926.

Necropsy was not obtained.

Case 5. A man with mitral stenosis and insufficiency, tricuspid insufficiency, severe myocardial failure, jaundice of the upper part of the body and general dependent anasarca. Died.

History. A white man, aged 27, who was admitted to Hospital on December 31, 1925 , giving a history of shortness of breath beginning three years previously. During these three years he had had repeated periods of failing circulation which would improve on rest in bed. In October, 1925, the present exacerbation began with severe dyspnea and palpitation on the slightest exertion and with edema of the feet and legs. In November, 1925, the abdomen became enlarged and two weeks before admission he noticed swelling of the wrists and fore-arms. There was a history of rheumatic fever in 1914.

On examination the patient was found to have signs of circulatory failure with mitral stenosis and insufficiency and auricular fibrillation. The liver was $6 \mathrm{~cm}$. below the costal margin and the spleen was just palpable. There was marked cyanosis of the nose, lips and cheeks, no pulsation of the veins in the neck or of the liver. Examination of the blood showed a Wassermanin reaction to be negative and there was no nitrogen retention. The van den Bergh reactions were both negative. There was no anemia. The electrocardiogram showed auricular fibril- 
lation with numerous ventricular extrasystoles sometimes appearing as pulsus bigemini, while at other times the extrasystoles occurred in groups of three or four in succession. With rest in bed and suitable treatment he improved somewhat, but on January 16th there was a sudden onset of increased dyspnea and cyanosis, with the appearance of a positive pulse in the veins of the neck and pulsation of the liver, and a loud to-and-fro murmur was to be heard at the lower end of the sternum. On January 20th there was an obvious icteroid tinge to the face, sclerae and upper part of the body above the nipples. The edema which had been constantly present since admission extended to the line of pigmentation. The ascites increased rapidly and on repeated occasions aspiration was required. Examination of the blood revealed a positive direct and indirect van den Bergh reaction and there were bile pigments in the urine. These were confirmed on repeated occasions but at no time was there a positive reaction in the ascitic fluid although a very faint indirect reaction was sometimes found. His condition continued practically unchanged except that the jaundice gradually became more and more pronounced, although occasionally there appeared to be remissions when the signs of tricuspid insufficiency .were not so conspicuous. On March 12th petechial hemorrhages became evident on the upper surface of the fore-arms and chest and the patient died on March 28th.

Necropsy was not obtained.

Case 6. A man with severe myocardial failure, mitral insufficiency, auricular fibrillation, dependent anasarca and jaundice of the upper part of the body. Died.

History. A white man, aged 32, who had been admitted to Hospital on one previous occasion, May 10,1923, with similar complaints. He had had two attacks of acute rheumatic fever previously. After his discharge from Hospital he was unable to work but continued in fairly good health until two weeks before admission when the symptoms of circulatory failure suddenly became acute. There was pronounced edema of the feet and legs which extended to the back, with swelling of the abdomen, which swelling was most pronounced on the right side where there was acute abdominal pain and tenderness. A week later it was noticed that he had jaundice of the face, sclerae and upper part of the body. This was still present when he was admitted to the Hospital on January 6, 1926.

On examination the patient was found to have signs of severe circulatory failure with mitral insufficiency and auricular fibrillation. There was edema of the legs, back and abdomen, with pronounced ascites. The liver was enlarged and pulsating while the veins of the neck showed a positive pulse. There was jaundice of the upper part of the body, face and sclerae, and bile pigments were present in the urine. The blood showed a negative Wassermann reaction and considerable nitrogen retention. There was both a direct and indirect van den Bergh reaction, while in the ascitic fluid both reactions were negative. There was some anemia $(3,830,000$ red cells, 75 per cent hemoglobin, and 14,000 leukocytes). The patient's condition became progressively worse and he died on January 17, 1926. 
Necropsy findings. Necropsy revealed chronic productive (sclerotic) endocarditis of the mitral valve causing mitral stenosis, hypertrophy and dilatation of the heart, productive pericarditis, ascites, hydrothorax, general anasarca and marked venous stasis of the liver, spleen and kidneys.

\section{CAUSATION OF THE PIGMENTATION}

The cause of jaundice in circulatory failure has been from time to time the source of some discussion. This discussion has arisen from the difference of opinion as to whether the hepatic lesion was due to a circulatory disturbance or whether a toxic factor-bacterial or nonbacterial-was responsible. Attention was first directed to the hepatic condition in these cases by Oertel (2) in 1904, when he described a lesion which he called multiple non'-inflammatory necrosis of the liver with jaundice in chronic cyanosis. In brief he found that the process consisted of a multiple, irregular, circumscribed solution of the liver cells, without parenchymatous degeneration or coagulation necrosis, and associated with a corresponding blood and bile stasis in the affected areas. In 1906 he described (3) the pathological anatomy of three more cases and in 1910, a fifth case (4). Oertel came to the conclusion that it was not a hepatitis but was most probably due to a mechanical cause in the form of chronic stasis. Mallory (5) on the other hand held that the disappearances of the liver cells in passive congestion of the liver was the result of bacterial necrosis. Bolton (6), in experimental passive venous congestion of the liver, came to the conclusion that the mechanical factor of stasis was the only cause in these cases.

More recently Keefer and Resnik (1925) (7) have again drawn attention to the appearance of jaundice in severe circulatory failure. They have drawn attention to the close association of pulmonary infarction with the subsequent appearance of icterus. They found in the ten cases which they have reported an apparently close relation between the appearance or the increase of icterus a few days after the occurrence of a pulmonary infarct. It appeared to them that this association might be caused by the increase of the anoxemia due to the well-known respiratory disturbances accompanied by anoxic anoxemia which follows pulmonary emboli.

In the nine cases which came to necropsy these workers found, 
apparently, a condition of the liver almost identical to that originally described in 1904 by Oertel (2). Given chronic passive congestion of the liver due to myocardial insufficiency, they adopted the following hypothesis: "It is possible that anoxemia, which is caused by pulmonary and circulatory impairment, resulting from pulmonary infarction, may depress the excretory function of already damaged liver cells to such an extent that jaundice appears." In order to test this hypothesis they carried out a series of experiments on dogs with suitable controls (8). The technique of the experiments in brief was as follows: to introduce into the stomach of the $\operatorname{dog} 4 \mathrm{cc}$. of carbon tetrachloride per kilo of body weight, and after a period of twenty-four hours to produce acute anoxic anoxemia by having the animal breathe nitrogen or a low oxygen mixture. Their result led them to conclude "that anoxemia may not only impair the function of the already damaged liver, but it may actually be responsible, at least in part, for the damage." Such a conclusion would seem quite justifiable from their experiments; but, these experiments are not a reduplication of the conditions found in the liver in cases of severe circulatory failure, particularly in those instances where tricuspid insufficiency is present.

It is a common observation that enlargement of the liver is one of the earliest signs of circulatory failure. In fact it may be present before edema of the more dependent parts is detectable. The reason for this is probably to be found in the fact that the liver is the only organ in which the greater part of the blood going to it has already passed through a capillary bed, namely that in the bowel. It might be supposed, therefore, that stasis due to a slowing of the circulation would be more easily produced in the liver than elsewhere. If this were carried to an extreme degree, interference with the bile secretion might be produced which would be probably further aggravated by anoxic anoxemia. Under such conditions it would not be expected that evidence of an obstructive jaundice would be found, but more likely that the blood pigments would be of the type found in hemolytic jaundice where an indirect van den Bergh reaction only is obtained. In fact this is the mild type of jaundice found so frequently in circulatory failure without pronounced tricuspid insufficiency.

The histological picture in the cases here reported was not that of simple passive congestion or nutmeg liver. The engorgement was 
more extreme and the destruction of the liver cells more complete. The engorgement of the smaller bile ducts was also a conspicuous feature while the engorgement of the hepatic vessels about the periphery of the lobules was greater than could be accounted for by simple venous stasis. In four of Oertel's cases and in all of those here reported, tricuspid insufficiency with positive pulsation in the veins of the neck and pulsation of the liver was present. It was strongly suggested that this factor of active increase of venous pressure in these veins and in the liver by transmission from the right ventricle is a most important factor in the production of this hepatic condition.

The manner in which the jaundice occurs would seem quite obvious from the histological appearance of the lesion. In the cases here reported there was direct evidence that the jaundice was due to an obstructive cause in that a positive van den Bergh reaction was always found. On the contrary in no case without tricuspid insufficiency was a positive direct van den Bergh reaction found, although in six cases with circulatory failure uncomplicated by dilatation of the right ventricle an indirect van den Bergh reaction was present. A further study of cases of circulatory failure has confirmed this original finding.

\section{DISTRIBUTION OF THE PIGMENTATION}

The distribution of the biliary pigmentation was confined to the head and upper parts of the body. In all of the cases there was pronounced anasarca extending up to approximately the level of the nipples. The hands and lower fore-arms were usually without pigmentation, although it was often present to some extent above the elbows. The pigmentation did not appear in the skin over areas where the edema was present. Indeed this was the most striking feature in the appearance of these cases. The skin over the edematous areas was, if anything, paler than usual while in the non-edematous areas a deep yellow to greenish-yellow pigmentation was present. The line of demarcation was quite clear cut. The transition zone from the pigmented to non-pigmented areas was only 2 to $3 \mathrm{~cm}$. broad.

In those cases where an autopsy was procured the endothelium of all the arteries and veins was deeply stained with bile pigments. This was followed into the smallest vessels possible. This indicated that the plasma of the blood passing through the vessels contained bile 
pigments although the skin was apparently not affected. In order to further determine this point blood was drawn from a vein in the leg by venesection in a case with pronounced edema of the lower extremities, and this plasma was also found to be deeply bile stained, although the edema fluid from the surrounding tissues was quite clear.

\section{EXAMINATION OF EDEMA FLUID}

$a$. Bile pigments. The dissociation of the edema and biliary pigmentation made an examination of the edema fluid imperative. The edema fluid was withdrawn either by means of cutaneous puncture with a three-cornered needle or through Southey's tubes. Every precaution was taken to prevent contamination with blood. In those cases where the surface tension was estimated precautions were taken to prevent the fluid from being mixed with cutaneous secretions.

In table 1 will be seen the comparative findings of biliary pigment, as estimated by the van den Bergh reaction, in both the serum and edema fluid. The cases 1 to 6 are those outlined in the protocols. In all there was both a direct and an indirect van den Bergh reaction in the serum while in only three of them was there an indication of any biliary pigments in the edema fluid. In two of these (cases 1 and 5) the indirect reaction was faintly positive. In Case 2 a faint direct reaction developed as well. At this time the greater part of the edema had been drained from the legs by means of Southey's tubes and edema fluid could only be obtained by massaging the legs over large areas surrounding the points of insertion of the tubes.

The almost constant absence of biliary pigments in the fluids of edema and ascites would point strongly to the conclusion that for some reason the capillary walls were impermeable to them. If this had not been the case it was to have been expected that the relative amounts of pigment would have been approximately the same in both fluids. In order to determine whether this was an isolated difference more extensive examinations of the serum and edema fluids were undertaken.

b. Serum proteins. These were not fractionated but the total quantity was estimated. This was done by means of a Zeiss dipping refractometer. In the serum the total proteins averaged 7.43 per cent while in the transudates they averaged 1.147 per cent. It was found, however, that the concentration was not equal in samples taken from 


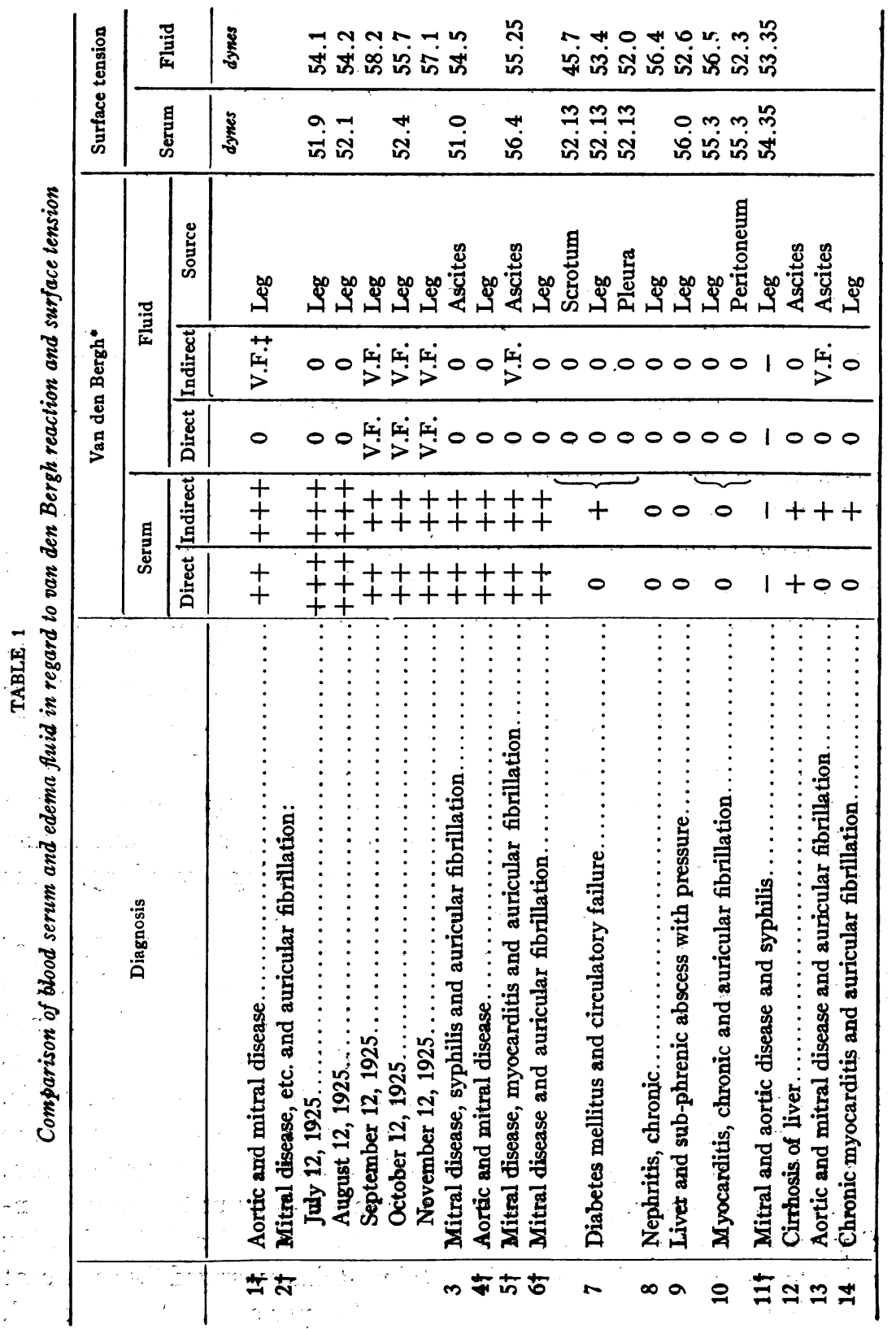


JONATHAN MEAKINS

145

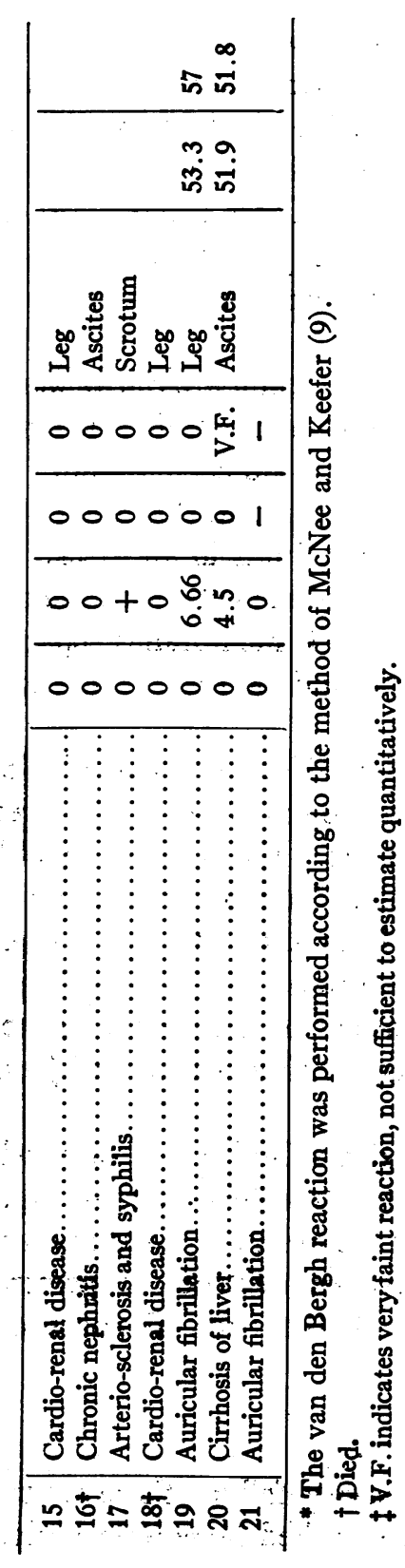


different regions. In six cases where the fluid was obtained from a serous cavity (peritoneum or pleura) the concentration on the average amounted to 2.057 per cent (varying from 0.97 to 3.64 per cent). In only two of these was the concentration of proteins in the serous fluid greater than 1.5 per cent and in both of these (Cases 5 and 13) there was a very faint van den Bergh reaction found in the fluid. The fluid obtained from the subcutaneous tissues had a comparatively low protein content, the average being 0.465 per cent.

c. Crystalloids. A further study was made to compare the amounts of sodium, potassium, magnesium, calcium, non-protein nitrogen, urea, creatinin and uric acid in the blood serum and edema fluid. In so far as these substances were concerned it was found that there was practically a normal balance between the two fluids in all cases. It was considered, therefore, that there was no interference with the diffusion of these substances through the permeable membranes separating the blood stream from the tissue spaces and cavities. This confirms the findings of previous workers.

$d$. Bile salts. In view of the dissociation of biliary pigmentation and edema it was deemed advisable if possible to determine the presence or absence of bile salts in these fluids. We had no reliable chemical method for estimating these salts. In spite of the finding of du Noüy that it required relatively large quantities of sodium glycocholate or taurocholate to effect the surface tension of serum it was decided to make comparative observations of the surface tension of the blood serum and edema fluid of cases with anasarca and jaundice, and also of those without jaundice.

The surface tension of the serum and edema fluid in ten cases will be found in the table. It was found that the differences were so slight as to be possible of explanation by other factors than a variation in the concentration of bile salts. We have no evidence at present, therefore, to lead us to a conclusion as to whether such salts pass into the edema fluid or not.

\section{SUMMARY}

It would seem probable that in circulatory failure there are two types of jaundice, one analogous to the so-called hemolytic variety where there is either an excess of the precursor of the bile pigments 
produced in the spleen or an inability on the part of the liver to transform the precursor into bile pigments. Such cases are those giving an indirect van den Bergh reaction in the blood serum. It is in this type that any increase in the anoxemic state of the liver would be expected to accentuate the impairment of hepatic function and thus promote an increase of the jaundice. On the other hand there is the obstructive type of jaundice where, although the bile pigments are formed from their precursor and secreted into the bile capillaries, they are prevented from passing to the larger ducts. The bile pigments (in their completed form which is different from their precursor) are then apparently reabsorbed into the hepatic circulation. In such cases it would be expected that the direct as well as the indirect van den Bergh reaction would be positive. This is what occurred in the cases reported above.

The manner in which jaundice is produced in circulatory failure would seem reasonable of explanation. Its peculiar distribution and the absence of the pigments in edema and ascitic fluid is not so clear. Two hypotheses present themselves; (1) that the permeable membrane between the capillary blood stream and the tissue spaces is impermeable to bile pigments, and (2) that there is such a gross interference with the blood flow through the tissues in circulatory failure that the contents of the tissue spaces remain uninfluenced by changes in the character of the blood plasma. If the second hypothesis were the correct one it would be expected that there would be a lack of equilibrium in other substances. This has been found to be the case only in regard to the proteins. It is suggested therefore that an important determining factor in establishing this equilibrium is the size of the molecule. In other words that the membrane between the blood and the tissue spaces is impermeable to substances with as large a molecular structure as proteins and bile pigments. This, however, is not absolutely the case in that edema-fluid although poor in proteins, as compared to the blood plasma, still does contain them to a moderate degree and at times in a concentration of 50 per cent of the plasma.

The presence of bile pigments varies in different secretions. They are found in the urine but not in the spinal fluid, ${ }^{3}$ milk or saliva. The

${ }^{3}$ In children bile pigments may be found in the spinal fluid and the meninges may be stained, but this has never been reported in adults. Further bile pigments and bile salts have been found in the contents of abscesses in cases of jaundice. 
reason for this selective action is not clear. On the other hand the pigmentation of the skin in jaundice would appear to be due to the disposition of bile pigments in the cellular elements rather than to a diffusion of the pigments into the inter-cellular spaces. Microscopic examination of the skin and other tissues shows the cells themselves to contain deposited bile pigments. If the bile pigments were not in solution in the inter-cellular spaces it would explain the isolated finding that jaundice of the skin may occur in edematous areas provided the jaundice antedates the occurrence of the edema. In this one instance the skin was pigmented while the edema fluid was free of bile pigments.

It would appear that with our present knowledge an adequate explanation of the absence of bile pigments in edema and serous fluids is not yet possible.

\section{BIBLIOGRAPHY}

1. Meakins, J., Can. Med. Ass. Jour., 1925, xv, 402. A Case Showing Unusual Distribution of Icteroid Pigmentation.

2. Oertel, H., Jour. Med. Res., 1904, xii, 75. Multiple Non-Inflammatory Necrosis of the Liver with Jaundice.

3. Oertel, H., Jour. Exper. Med., 1906, viii, 103. A Further Contribution to the Knowledge of Multiple Non-Inflammatory Necrosis of the Liver with Jaundice. (Hepar necroticum cum Ictero) and to the Knowledge of Cell Degeneration and Cytolysis in General.

4. Oertel, H., Arch. Int. Med., 1910, vi, 293. Multiple Non-Inflammatory Necrosis of the Liver with Jaundice in Chronic Cyanosis.

5. Mallory, F. B., Jour. Med. Res., 1911, xxiv, 455. Chronic Passive Congestion of the Liver.

6. Bolton, Chas., Jour. Path. \& Bacter., 1914, xix, 258. The Pathological Changes in the Liver Resulting from Passive Venous Congestion Experimentally Produced.

7. Keefer, C. S., and Resnik, W.H., Jour. Clin. Invest., 1926, ii, $375 . \quad$ Jaundice Following Pulmonary Infarction in Patients with Myocardial Insufficiency: I. A Clinical Study.

8. Resnik, W. H., and Keefer, C.S., Jour. Clin. Invest., 1926, ii, 389. Jaundice Following Pulmonary Infarction in Patients with Myocardial Insufficiency: II. An Experimental Study.

9. McNee, J. W., and Keefer, C. S., Brit. Med. Jour., 1925, ii, 52. The Clinical Value of the van den Bergh Reaction for Bilirubin in Blood. 